\title{
La plasticidad forzada. Cuerpo y trabajo
}

\author{
Forced plasticity. Body and Work
}

PABLO LÓPEZ ÁLVAREZ*

\begin{abstract}
Resumen: Las transformaciones en la organización del trabajo son esenciales para una filosofía contemporánea del cuerpo, aunque no sea un campo prioritario en los estudios actuales. Este escrito expone el sentido de alguna de estas transformaciones, relativas a la organización espaciotemporal del trabajo, la semántica de la nueva cultura del trabajo y la individualización de los procedimientos de evaluación.

Palabras clave: trabajo, cuerpo, flexibilidad, postfordismo.
\end{abstract}

\begin{abstract}
Transformations in the organization of work must be crucial for contemporary philosophy of body, although current studies may not consider this issue as critical. This paper focuses on the nature of some of this transformations, concerning the spatial and temporal organization of work, the semantics of new work culture and the individualization of evaluation processes.
\end{abstract}

Keywords: work, body, flexibility, postfordism.

En una anotación de la Filosofía del derecho (§ 48), relativa a los derechos de la personalidad y la naturaleza corpórea del individuo, Hegel escribe: puesto que «soy viviente como libre en un cuerpo [...] no se debe abusar de esta existencia viviente hasta hacerla una bestia de carga»; «mientras vivo [...] mi cuerpo es la existencia de la libertad y yo siento en él». Sin duda, cada sujeto puede imaginar que es libre a pesar del cuerpo o frente al cuerpo, puede negarlo o afligirlo. Sin embargo, para los otros es en su cuerpo: «sólo soy libre para los otros si soy libre en la existencia». En definitiva, y más claramente: «la violencia ejercida por otros sobre mi cuerpo es violencia ejercida sobre mí».

El texto es complejo y no se pretende aquí comentarlo por extenso. Se trata de destacar un punto de lo que Hegel expone. A la luz de su posición, los derechos del cuerpo poseen un estatuto propio, independiente de la voluntad del individuo. El cuerpo ha de ver reconocido su derecho al margen de la decisión «subjetiva». Como cuerpo, es indisponible, y

Fecha de recepción: 10/06/2016. Fecha de aceptación: 06/09/2016.

* Profesor titular de Filosofía en el Departamento de Historia de la Filosofía, Estética y Teoría del Conocimiento de la Universidad Complutense de Madrid. Correo: pla@ucm.es. Trabaja en los campos de la historia de la filosofía moderna, la filosofía política y las teorías críticas contemporáneas. Recientemente ha publicado una edición bilingüe de Sobre la necesidad y la necesidad, de Thomas Hobbes (2015) y ha participado en el volumen La actualidad de Michel Foucault con el artículo «Sigue cierta algarabía. Foucault, el neoliberalismo y nosotros» (2016). 
es indisponible en primer lugar para el sujeto propietario. Es una «cosa», ciertamente, pero con un estatuto peculiar: es una cosa excluida de las relaciones de comercio [res quarum commercium non est]. Retomando algunos elementos del derecho romano, Hegel muestra los límites de las metáforas del cuerpo-máquina y el cuerpo-propiedad, y piensa el cuerpo como realidad física, como conjunto de hábitos teóricos y prácticos y como institución jurídica. Es la base para una concepción normativa del cuerpo.

Quisiera apoyarme en esta idea, fundamental en el tratamiento filosófico del cuerpo en el primer capitalismo, para pensar algunas realidades actuales, que culminan ese proceso que Hegel vio ascender y temió. Me refiero a los modos de organización del trabajo y las culturas laborales desarrolladas en las últimas décadas, en el contexto del capitalismo financiero o neoliberal, la forma en la que redefinen la relación entre cuerpo y subjetividad, y los nuevos problemas que plantean para pensar el estatuto del cuerpo y la violencia ejercida sobre él.

Las últimas décadas han asistido a un aumento extraordinario de los estudios sobre el cuerpo, desde perspectivas muy diversas. Sería imposible intentar enumerarlas. Esta proliferación de análisis coincide en el tiempo con el despliegue de una nueva configuración histórica del capitalismo, que en medida importante se ha consolidado a través de reestructuraciones en el campo del trabajo y las relaciones laborales. Es llamativo, por ello, que los estudios en torno al cuerpo se hayan alejado de los problemas del trabajo, y que éste, en general, haya perdido presencia en la filosofía contemporánea. La importante Historia del сиerpo, dirigida por Corbin, Courtine y Vigarello (2005), apenas dirige atención al trabajo en su tercer volumen, dedicado al siglo XX, aunque sí lo hace en el anterior, que recorre el siglo XIX. En otro valioso proyecto de referencia, Fragmentos para una historia del cuerpo humano, editado por Michel Feher, el trabajo se encuentra igualmente ausente. En el índice de materias de su extensa bibliografía sobre historia del cuerpo, situada al final del tercer volumen, se buscará en vano el término «trabajo» entre otras referencias mucho más frecuentadas: ayuno, cadáver, desnudez, enfermedad, higiene, melancolía, monstruos, piel, resurrección, sangre, sexualidad. Se diría que el trabajo se ha vuelto irrelevante para la consideración del género, el sufrimiento o la salud desde la perspectiva de una historia del cuerpo. Del mismo modo, sólo la elusión del trabajo (y la clase) parece justificar la fantasía postcorpórea de Le Breton en Adiós al cuerpo: «nunca antes, sin duda, como sucede en nuestras sociedades occidentales, los hombres habían utilizado tan poco su cuerpo, su movilidad, su resistencia. La tensión nerviosa (estrés) ha rebasado el gasto físico. Las capacidades musculares caen en desuso fuera de los gimnasios, relegadas por la energía inagotable que proporcionan las máquinas [...] El anclaje corporal de su existencia pierde poder» (Le Breton, 2007, 23). ¿Hemos llegado a incorporar el discurso hegemónico según el cual la actividad laboral es cuestión de energías y disposiciones (y no tanto de cuerpos y necesidades)? ¿Se ha impuesto hasta ese punto la semántica de la empresa [enterprise] sobre el lenguaje -pesado, material- del trabajo [work]?

Quizá el proceso sea antiguo. Christophe Dejours ha señalado que ya en los años 70 el problema del sufrimiento laboral desapareció de la agenda de la izquierda europea. En ese espacio pudo desarrollarse una utopía alternativa, la neoliberal, que ofrecía una lectura propia de las relaciones entre subjetividad y trabajo, en los conceptos de la teoría del capital humano y los recursos humanos, la flexibilización de las jerarquías laborales, la innovación y el reconocimiento de los perfiles profesionales únicos. ¿En qué términos pensar la vio- 
lencia inscrita en formas de organización laboral que movilizan de un modo tan eficaz las exigencias de independencia y autoorganización? Para delimitar el alcance del problema, me centraré en tres rasgos de estas nuevas realidades laborales, a partir de algunos estudios específicos: la nueva percepción laboral del espacio y el tiempo, las mutaciones epistemológicas vinculadas a la nueva cultura del trabajo y la extensión de modelos de evaluación individualizada del rendimiento.

\section{El nuevo espíritu del trabajo}

Sergio Bologna ha estudiado en profundidad las variaciones organizativas que arrastra la extensión de la figura del trabajador autónomo de segunda generación. Algunos de sus más importantes escritos se encuentran recopilados en el volumen Crisis de la clase media y postfordismo (2006). Bologna analiza las aproximaciones teóricas al trabajo autónomo en las primeras décadas del siglo XX, y en particular en el escenario de Weimar (Lederer, Speier, Geiger, Kracauer). Es el momento de la difusión del paradigma fordista-taylorista de producción, y del tránsito, por tanto, del trabajo por cuenta propia al trabajo asalariado, vinculado directamente con la retórica de la «modernización» de las relaciones sociales y que habría de convertirse en la base real para la ciudadanía en los países avanzados. La transición del fordismo al postfordismo a partir de la década de 1970 invertirá aquel camino, para discurrir desde las formas del trabajo por cuenta ajena a la construcción de una sociedad de trabajadores no asalariados (en condiciones muy diferentes a las del trabajo autónomo de los años 20 y 30).

Procedente de la tradición intelectual y militante del operaísmo italiano, y experimentado igualmente en el trabajo por cuenta propia, Bologna acentúa de entrada el nexo entre la crisis del modelo productivo fordista y el despliegue de iniciativas sociales que ensayan formas alternativas de existencia. La confianza en los propios recursos sociales y relacionales (no tanto profesionales) de las generaciones que protagonizaron y heredaron las demandas del 68 fortalece el rechazo a la inserción en organizaciones jerarquizadas y el desafío a los hábitos de pensamiento que ven en el trabajo asalariado la forma natural de integración social y de adquisición de derechos de ciudadanía. Las críticas a la gestión institucional de las técnicas y las disciplinas científicas tradicionales enlazan con el despliegue de prácticas sociales descentralizadas y autoorganizadas, y con formas diversas del trabajo autónomo. La demanda de independencia sobrepasa completamente la cultura fabril y sus tipos sociales característicos, y se concreta en la extensión de iniciativas cooperativas (más bien que de empresas unipersonales o microsociedades). En el entorno alemán, las variedades del trabajo por cuenta propia se inscriben de manera natural en los espacios del ecologismo radical, las luchas antinucleares, los proyectos de pequeñas unidades de producción agrícola, el feminismo y el movimiento libertario. En amplios grupos sociales, la libre iniciativa laboral se impone como alternativa al trabajo asalariado en la administración pública o en el aparato productivo alemán, sometido entonces a un profundo proceso de reestructuración y desindustrialización.

Una parte importante de las actuales aproximaciones al neoliberalismo está marcada por la utilización de una lógica binaria, que acentúa el relato de la derrota del Estado social europeo a manos de la new economy y limita su imaginación política a la rehabilitación del modelo institucional y los hábitos de pensamiento entonces vencidos. Por lo general, esta 
posición minimiza la magnitud de las luchas sociales y laborales desarrolladas en aquellos años, que desafiaban los equilibrios del capitalismo pactista y el imaginario industrial-laboral fordista, y planteaban expectativas fuertes de democratización, bienestar y repolitización de la existencia social. En este contexto, es prudente tomar en consideración la exposición de Bologna:

El profundo impulso ético, la fuerte curiosidad intelectual y la disponibilidad a la experimentación crearon un hábito, una auténtica mentalidad colectiva, un estilo de vida característico. Era una forma mentis que, aunque no se pueda parangonar con la ética protestante de la que habla Weber, sin duda tuvo un efecto no muy distinto en la creación de energías emprendedoras y en la formación de una «ética del trabajo» distinta y que, por lo tanto, no cabe banalizar o ridiculizar. Si, en lugar de mirar al fenómeno con los ojos de quienes esperaban una palingenesia que luego no tuvo lugar y, desilusionados, destruyen con furia iconoclasta su propio pasado, se mirase a la generación de los «alternativos» simplemente como a un grupo de personas que, bien o mal, intuyó que sólo «un nuevo modo de trabajar» permite a los débiles, es decir, a las microempresas, mantenerse en el mercado y sobrevivir, se tendría probablemente una visión más equilibrada del fenómeno que se produjo a caballo entre las décadas de 1970 y 1980, por lo que sabemos de él, no sólo en Europa, sino también en Norteamérica. (Bologna, 2006, 48-49).

\section{Espacio, tiempo, retribución}

La definitiva superación del trabajo asalariado como forma esencial de la ciudadanía a lo largo de la década de 1990, correlativa a la caída de los regímenes del socialismo real, permite medir las consecuencias del proceso y las figuras reales en las que se ha encarnado el trabajo autónomo. Los modelos de la subcontratación [subcontract], el autoempleo [selfemployment], el trabajador autónomo [freelance] y el trabajador asociado [associate employee] definen una condición laboral característica, que puede estudiarse desde el punto de vista de sus patrones materiales y corporales. Un importante artículo de Bologna, «Diez tesis para la definición de un estatuto del trabajador autónomo», inicialmente incluido en Il lavoro autonomo di seconda generazione. Scenari del postfordismo in Italia (1997) y completado años más tarde con «La undécima tesis» (2005), explora algunas de estas dimensiones. En primer lugar, la percepción del espacio se ve afectada por la desestructuración de la organización espacial tradicional del fordismo (fabrica/oficina). La importancia vital del «lugar» de trabajo es erosionada por un conjunto de mutaciones que superan la distinción tradicional entre el espacio privado y el espacio de trabajo, con sus normas y culturas perfectamente diferenciadas. La domestificación u hogarización del trabajo [domestication] rompe las antiguas fronteras entre hogar y fábrica, vida privada y vida laboral, entre los ritmos afectivos y los ritmos del trabajo. No se trata exclusivamente de que el trabajo se realice en el domicilio, sino de que en medida creciente los hábitos y las reglas privadas del trabajador definen y permean su desempeño laboral: el propio espacio del distrito industrial adquiere una forma característica, abierta a normas mixtas y (potencialmente) a patrones diferentes de cooperación y organización. Se produce una intensa movilización laboral de cuerpos 
capaces de conducir simultáneamente producción y reproducción, a partir de patrones temporales flexibles y múltiples, espacios híbridos de trabajo en los que rige una altísima tasa de explotación y lógicas de dominación no controladas sindicalmente.

El cuerpo se vuelve productivo por su mera existencia, y en estas nuevas condiciones la feminización del trabajo es un efecto de particular importancia, como ha subrayado recientemente Cristina Morini (2014): no se alude con ello únicamente al aumento de la presencia femenina en el mercado de trabajo, sino también a la generalización de aquellas condiciones de explotación históricamente ejercidas sobre las mujeres. La máquina que la biopolítica neoliberal quisiera reproducir y replicar es -sin ninguna duda- el cuerpo femenino (nódulo de producción y cuidados, saberes prácticos y competencias relacionales, movilidad y adaptación).

El trabajo de las mujeres parece representar un modelo al que el capitalismo contemporáneo mira con creciente interés, tanto en todo lo relacionado con las formas (precariedad, movilidad, fragmentación, bajos niveles salariales), como en lo relativo a los contenidos, considerada la nueva centralidad de la explotación intensiva de la cualidad, la capacidad y los saberes individuales. Las mujeres no sólo son funcionales a un mercado de trabajo flexible, tanto en términos de entrada como de salida, según las exigencias productivas y sociales del momento, sino que condensan también en sí, en un único cuerpo, la posibilidad de asumir los roles productivo y reproductivo. (Morini, 2014, 83-84)

La percepción del tiempo se ve también alterada, hasta el punto de constituir un rasgo esencial del nuevo trabajo autónomo. De entrada, se produce la supresión del límite de la jornada de trabajo, característica de la regulación laboral fordista, y desaparece con ello uno de los clásicos planos de conflictividad del trabajo asalariado, vinculado a la comprensión del tiempo como modo de dominación (Postone). Las utopías del final del trabajo, de la reducción de la jornada laboral por la automatización de la producción y de la emergencia de las sociedades del ocio, han envejecido a una sorprendente velocidad. Es cierto que la medición estadística del fenómeno encuentra problemas e inercias: la referencia para la cuantificación de la jornada sigue siendo el trabajo asalariado en grandes grupos privados o públicos, y los marcos contractuales no permiten capturar la organización real del trabajo por cuenta propia. En todo caso, la ampliación de la jornada laboral es un hecho, paralelo al incremento de la productividad y a la intensificación de los ritmos de trabajo. La vida del cuerpo se moviliza sin resto en el tiempo de trabajo, incluyendo los aspectos, no reconocidos económicamente, del establecimiento de relaciones y de comunicación. Al contrario de lo que ocurre en la función pública o el trabajo asalariado, la actividad del trabajador autónomo no sale jamás del mercado y el sujeto ha de mostrar una flexibilidad infinita para adaptarse a sus exigencias. El tiempo se percibe bajo la forma del riesgo (la amenaza de la falta de trabajo) y ello impide la distribución anticipada del esfuerzo por segmentos de tiempo: la planificación a largo plazo es inviable, del mismo modo que la adaptación a los ritmos vitales de otros sujetos (fragmentación de las biografías laborales).

Finalmente, ello tiene consecuencias para la retribución del trabajo y para la pervivencia de la forma salarial nacida del industrialismo. Es característica del trabajo autónomo la rup- 
tura del nexo entre salario y unidad de tiempo (día, hora, minuto): lo que se retribuye o se «factura» es un servicio o una prestación, en plazos temporales abiertos a la fragmentación y la incertidumbre. El valor central de la axiología del trabajo flexible no es el tiempo del trabajador, sino su energía: han de desarrollarse técnicas para aumentar la propia energía, recuperarla y renovarla, en condiciones de tiempo finito. Pero esto supone una transformación insólita en el interior de la relación salarial. El salario había implicado siempre la garantía por parte del empleador del mínimo vital suficiente para el mantenimiento de la energía laboral del trabajador. El Estado social, acogiendo las tradiciones cooperativas y sindicales, añadiría la protección del trabajador en condición de desempleo, enfermedad o inhabilitación, así como la consolidación del salario indirecto en la forma de consumo colectivo. En el marco de la nueva economía, por el contrario, «se ha sancionado por primera vez de facto el principio de que la subsistencia de la fuerza de trabajo ya no es un problema del que el empleador o el Estado deban hacerse cargo» (Bologna, 2014, 72). Las relaciones sociales dejan de tener como problema constitutivo la reproducción de los cuerpos. ¿Es ésta otra de las consecuencias invisibles del tránsito de la centralidad del trabajo a la de la empresa: el eclipse del compromiso con la subsistencia ajena en beneficio de un generalizado riesgo existencial?

Como mínimo, este hecho se articula bien con otra modificación esencial, la desjuridificación laboral. La consolidación del derecho del trabajo en los órdenes legislativos contemporáneos sólo fue posible por el reconocimiento de una asimetría de poder entre los agentes que intervienen en la compraventa de la fuerza de trabajo. El derecho laboral es un modo de limitar, contra la libertad mercantil, los efectos de dominación que se siguen de esa asimetría. En la medida en que el pensamiento neoliberal plantea esta relación como una relación comercial entre empresas, las estructuras de poder desaparecen a la mirada (Alonso, 2009). Ello sólo puede significar la destrucción de los derechos consolidados a través de la negociación y el conflicto, al tiempo que la organización objetiva del trabajo cierra los tradicionales cauces de acción colectiva: asociación, delegados sindicales, huelga. En ausencia de formas alternativas de garantizar la protección y la subsistencia material, lo que se juega en este proceso es el vaciamiento de sentido del Estado social de derecho, al menos en tanto que éste ancla su génesis en el principio de solidaridad y codependencia de los cuerpos. En 2016 el derecho del trabajo está en discusión en la socialdemocracia europea. ¿Cómo ha llegado este aspecto a parecer lateral a una historia política del cuerpo?

El diagnóstico de Bologna se esfuerza por mostrar la naturaleza ambivalente de las mutaciones en el campo del trabajo, que evita juzgar «de manera exclusivamente negativa, como intensificación de la explotación e inseguridad permanente» $(2014,71)$. El trabajo autónomo se liga a formas de autoorganización temporal y de relación con las instituciones que acogen un sentido diferente de la libertad. En todo caso, sus estudios obligan a reconocer las dimensiones corpóreas de la nueva cultura laboral y previenen contra la retórica del trabajo inmaterial:

Es probable que no exista actividad humana donde la fisicidad, el tesón, el esfuerzo, el sentido de la disciplina, la adaptación al ambiente y la flexibilidad estén sometidas a un apremio mayor que durante el ejercicio de una función laboral. No existe actividad de la que dependa en mayor medida la reproducción física del individuo 
[...] El trabajo, de por sí, encierra la quintaesencia de la materialidad; definirlo como «inmaterial» es absurdo y da lugar a equívocos. De hecho, estos teóricos del «trabajo inmaterial» dedican una atención muy exigua o nula a lo que constituye el fenómeno más significativo del postfordismo, a saber, la extensión de la jornada laboral social; la misma omisión se produce respecto a las transformaciones de la forma salarial y, por lo tanto, respecto a la retribución del trabajo autónomo. Las mercancías pueden ser inmateriales, los trabajos no. (Bologna, 2006, 128)

\section{La persistencia de la disciplina: organización y sufrimiento}

Damián Pierbattisti ha destacado en La privatización de los cuerpos (2008) las mutaciones epistemológicas que arrastra el nuevo modelo laboral. Su pormenorizado análisis toma pie en el proceso de privatización de las empresas argentinas de telecomunicaciones de los años 90 , y en el tipo de constelación semántica que sirvió de base de legitimación. El nuevo vocabulario del trabajo gravita en torno a la figura del empresario de sí, estudiada por Foucault ya en 1979, e incide sobre todo en las capacidades de «auto-dirección» [self-steering capacities] de los sujetos (Vázquez, 2005). La valoración positiva de la inseguridad como «empleabilidad» ataca al empleo estable, y justifica la privatización de los riesgos corporales y la individualización e informalización de la relación laboral. Los modelos de contratación flexible y atípica se acompañan de reformas en el tipo de negociación laboral y de transformaciones en el sistema de la seguridad social. La empleabilidad implica el sometimiento de todo trabajador a instancias de examen continuo y a la exigencia de renovación permanente de las aptitudes: el trabajo se transforma en una zona de vulnerabilidad e inseguridad en la que el empleo ha de ser merecido a cada momento.

La sustitución del lenguaje del trabajo por el de la empresa supone el despliegue de todo un arsenal «ético», un nuevo ethos de empresa: son esenciales las transformaciones discursivas que realizan el tránsito de «usuario» a «cliente», de «empleo de por vida»a «empleabilidad» y de «trabajador» a «colaborador», así como la valoración fundamental de nociones como «pro-actividad», «autonomía responsable», «autocompetencia», «creatividad e iniciativa», «atención al cliente» «sacrificio», «adaptación» y «mentalidad». Cada uno de estos elementos tiene su traducción corporal, y está en la base de culturas del cuerpo socialmente extendidas. Son imprescindibles para el incremento del nivel de productividad y «la construcción de cuerpos extremadamente productivos y flexibles» (Pierbattisti, 2008, 59). El proceso, además, supone nuevas técnicas de estigmatización de los cuerpos: los cuerpos lentos, predecibles, vinculados a culturas laborales antiguas e ineficientes, han de ser políticamente neutralizados, por su escasa posibilidad de «reutilización» y su incapacidad para producir «ciclos cortos de rendimiento máximo» (Landa/Marengo, 2010). En cada uno de los procesos de privatización/modernización empresarial, es crucial inmunizarse ante el contagio de los cuerpos improductivos, distinguir lo viejo de lo nuevo, desplegar formas renovadas de compromiso y disponibilidad, limpiar, eliminar residuos, luchar contra la esclerosis y el anquilosamiento. La valoración de la disposición viril y bélica, de la capacidad de cometer violencia sobre el cuerpo dominado, es tan importante en este punto como en la lucha contra los competidores económicos. 
El psicopatólogo del trabajo Christophe Dejours ha estudiado con detalle algunos de estos elementos, y en particular la conexión entre los modos de organización del trabajo y la intensificación y banalización del sufrimiento laboral. Ya se ha mencionado su idea de que, en el marco de la crisis productiva y de beneficio del fordismo, las organizaciones políticas y sindicales de izquierda eludieron la consideración de los problemas de la subjetividad y el sufrimiento, centrando su fuerza reivindicativa en la defensa del empleo. El neoliberalismo supo metabolizar ese contexto e inscribir en él una utopía particular: aquella que se formulaba en el lenguaje de la identidad, la motivación y la cultura empresarial, y permitía la transición entre el trabajo como productor de dolor al trabajo como productor de autorrealización. Es un elemento constante en esta exposición.

Las investigaciones en psicopatología del trabajo emprendidas en la década de los setenta tuvieron que enfrentarse en su momento con la negativa de los sindicatos y la condena de la izquierda. Todo lo que estaba ligado a la subjetividad, el sufrimiento subjetivo, la patología mental y los tratamientos psicoterapéuticos suscitaba desconfianza, e incluso era negado públicamente [...] Cualquier aproximación a los problemas psicológicos por parte de psicólogos, médicos, psiquiatras y psicoanalistas estaba marcada por un pecado capital, el de privilegiar la subjetividad individual, conducir a prácticas individualistas y obstaculizar la acción colectiva [...] Se sospechaba que las preocupaciones sobre la salud mental, consideradas antimaterialistas, obstaculizaban la movilización colectiva y la conciencia de clase. (Dejours, 2009, 55).

Las consecuencias de esta posición son amplísimas. Por un lado, se produce una descalificación del problema del sufrimiento laboral, que contribuye a una mayor tolerancia del fenómeno pero también a un alejamiento de las vivencias de los trabajadores con respecto al lenguaje de las élites sindicales y el consiguiente debilitamiento de las organizaciones representativas. Por otro lado, se favorece una concepción del trabajo que lo entiende como intrínsecamente no problemático. El trabajo sería como tal una realidad transparente, carece de fricciones, y podría ser desarrollado potencialmente por agentes automatizados. La única dificultad asociada a él descansaría en su adecuada organización empresarial: de la turbiedad de la producción transitamos hacia la luminosidad de la gestión, un rasgo central de la mutación discursiva del neoliberalismo.

En este plano se inscribe un elemento al que Dejours presta particular atención: el despliegue de técnicas de medición y evaluación individualizada del rendimiento laboral. Dejours parte aquí de una tesis epistemológicamente fuerte: la imposibilidad de cuantificar con rigor la calidad del trabajo individual. Lo esencial del trabajo no descansa en la relación entre objetivos y resultados, sino en la capacidad para trascender lo prescrito en virtud de una particular implicación del cuerpo: por ello, ninguna medición científica puede apresarlo adecuadamente. Las cuidadoras de bebés enfermos que deciden tejer mientras vigilan el estado de los niños no se atienen a ninguna orden, y puede parecer que descuidan su tarea: al contrario, están colocando las condiciones para no ser vencidas por el sueño y mantener la escucha. Se trata de una inteligencia corporal que resulta difícil de exponer y que sobrepasa la conciencia que el trabajador posee de su tarea. En general, cualquier trabajo se arruina si el trabajador se limita a llevar a término lo establecido de antemano: 
«el trabajo es la actividad coordinada de hombres y mujeres para hacer frente a aquello que sería imposible lograr mediante la ejecución estricta de lo prescrito» (Dejours, 2009, 86).

Ignorando lo real del trabajo, el procedimiento de evaluación individualizada tiende a leer los fracasos en el trabajo como indicio de falta de competencia, seriedad o capacitación, en todo caso atribuible al trabajador, y sobre esta posición se articula el discurso en torno al factor humano como fuente de error. Cada trabajador es examinado no sólo por la conquista de objetivos y las tasas de eficiencia, sino también por su capacidad para actuar sobre sí mismo, sobre su propio cuerpo y sus capacidades: se calibra su motivación, su compromiso, su propensión al esfuerzo y la disciplina, su involucración (en cuerpo y alma). La evaluación continua produce competitividad generalizada entre los trabajadores, acentúa la presencia del miedo en las relaciones laborales, fragiliza las solidaridades laborales, imposibilita la acción colectiva, aumenta la presión productiva y hace al sujeto más vulnerable al acoso y a las patologías de sobrecarga laboral (burn out, karoshi, trastornos músculo-esqueléticos). Lo enigmático en estas condiciones no es la enfermedad mental, sino la normalidad misma. Obligaciones de tiempos, ritmo, formación, aprendizaje, nivel de conocimiento, rapidez en el desarrollo de habilidades intelectuales y prácticas: a partir de una cierta intensidad (cadencia) y una cierta extensividad (duración de la jornada), la actividad laboral entra en contradicción con las estructuras de la subjetividad corpórea, y la preocupación subjetiva esencial es si se podrá resistir el trabajo, psíquica y físicamente.

El sufrimiento laboral se ve incrementado en los sistemas individualizados de control por la necesidad de mentir constantemente sobre el propio trabajo, de elaborar relatos de justificación que puedan satisfacer los patrones de evaluación de la calidad pero que no recogen en ningún caso la realidad de la práctica laboral, sus dificultades y condiciones reales. ¿Qué ocurre cuando el elemento esencial del trabajo -las mediaciones corporales entre lo prescrito y lo realizado: la fatiga, el límite, la inteligencia del cuerpo- está ausente de los programas de evaluación? ¿Qué supone para el poder del trabajador la obligación de hablar un lenguaje formulado en patrones abstractos de calidad y rendimientos cuantitativos individualizados, ciego al trabajo como experiencia colectiva y cualitativa? (Deranty, 2011). La aplicación del lenguaje del management al trabajo no incrementa su calidad, pero es completamente funcional a las exigencias políticas del postfordismo: resta peso al conocimiento acumulado por el trabajador en su propia práctica y le arrebata el control sobre su trabajo (el conocimiento del gestor es muy superior al conocimiento del trabajador).

Dejours plantea un último aspecto, igualmente importante. La permanente negación del propio sufrimiento laboral, como estrategia de defensa del sujeto ante la amenaza de desestructuración psíquica y somática, tiene como efecto la incapacidad para captar el sufrimiento laboral ajeno. Las condiciones organizativas en las cuales el sujeto se cierra a su propio sufrimiento favorecen la banalización de la violencia en el plano laboral, la dificultad para aprehender ese sufrimiento como injusticia y movilizar contra ella la acción colectiva. La tesis de Dejours merece ser seguida en profundidad: la prioridad del sufrimiento sobre la violencia alude al hecho de que el miedo generalizado en las relaciones laborales produce en el sujeto una serie de reacciones defensivas, entre ellas la insensibilización a la violencia ejercida sobre trabajadores y no trabajadores. La reacción ante los suicidios en los centros de trabajo es elocuente en este sentido. La organización laboral del capitalismo neoliberal no puede entenderse sin la valoración positiva de la disposición viril a hacer lo que ha de 
hacerse -limpiar, evaluar, seleccionar cuerpos aptos-, el carácter fuerte, la crudeza y la capacidad para hacer un buen uso del dolor. Contra lo anticipado por Foucault, el neoliberalismo no prescinde del imaginario bélico en su retórica de legitimación.

Las anteriores consideraciones, presentadas programáticamente, quieren destacar implicaciones corporales de las nuevas formas de organización laboral (más que del trabajo mismo) que son esenciales para una filosofía del cuerpo contemporánea. Se juegan aquí, por una parte, los devastadores efectos de aplicar sobre la realidad finita del cuerpo la gramática de la energía infinita, la plasticidad ilimitada. El dominio del cuerpo a través de su desmaterialización es un elemento fundamental de la cultura corporal del neoliberalismo: el cuerpo se encuentra a disposición del sujeto, y por ello su rendimiento y su morfología pueden someterse a una evaluación moral en la que la responsabilidad del individuo es central. Por otra parte, este complejo proceso no puede leerse exclusivamente como un aumento de la explotación (ejercida sobre un sustrato material pasivo). Se articula desde su inicio con la exigencia de superación de las formas organizativas del trabajo asalariado y la disciplina fabril, y con luchas muy heterogéneas contra la rígida concepción fordista del trabajo, sus límites políticos y subjetivos. Ello invita a avanzar en planteamientos que eviten la pura expectativa del retorno y tracen una institucionalidad con poder de garantizar la dignidad material del cuerpo y al mismo tiempo su autonomía y potencial cooperación.

\section{Referencias}

Alonso, Luis Enrique (2009): Prácticas económicas y economía de las prácticas, Madrid, Catarata.

Bologna, Sergio (2006): Crisis de la clase media y postfordismo, Madrid, Akal.

Dejours, Christophe (2009): Trabajo y sufrimiento. Cuando la injusticia se hace banal, Madrid, Modus Laborandi.

Deranty, Jean-Philippe (2011): «Work and the experience of domination in contemporary neoliberalism», Actuel Marx, 49.

Landa, Inés; Marengo, Leonardo (2010): «Devenir cuerpo empresa. El nuevo capitalismo y sus tramas de sujeción», Actuel Marx / Intervenciones, 9.

Le Breton, David (2007): Adiós al cuerpo, México, La Cifra Editorial.

Morini, Cristina (2014): Por amor o a la fuerza. Feminización del trabajo y biopolítica del cuerpo, Madrid, Traficantes de sueños.

Pierbattisti, Damián (2008): La privatización de los cuerpos. La construcción de la proactividad neoliberal en el ámbito de las telecomunicaciones (1991-2001), Prometeo, Buenos Aires.

Vázquez, Francisco (2005): «"Empresarios de nosotros mismos”. Biopolítica, mercado y soberanía en la gubernamentalidad neoliberal», en J. Ugarte (ed.), La administración de la vida. Estudios biopolíticos, Barcelona, Anthropos. 